\title{
Interactive comment on "Impacts of the COVID-19 lockdown on air pollution at regional and urban background sites in northern Italy" by Jean-Philippe Putaud et al.
}

Jean-Philippe Putaud et al.

jean.putaud@ec.europa.eu

Received and published: 25 November 2020

We would like to thank Referee \#2 for constructive comments to our manuscript posted on 4 November, and we are glad to discuss those interactively.

1. We include below a pie-plot showing NOx and VOC 2015 sectorial emissions in Italy as derived from the EDGAR emission inventory. We would be glad to know if Referee \#2 thinks that a pie-plot showing SO2 emissions would also be useful. These pie plots will be included in the Supplement to our manuscript.

2. Regarding CAMS-Ensemble forecast description, we intended to include a para- 
graph as follows: "The CAMS Regional Air Quality forecast are routinely quality controlled and dedicated evaluation report are published on a quarterly basis for both individual and ENSEMBLE model. The relevant report for March/April/May 2020 is available at https://atmosphere.copernicus.eu/sites/default/files/202009/CAMS50_2018SC2_D5.2-3.1.ENSEMBLE-2020MAM_202008_NRTProduction_Report_v1.pdf. For the specific case of PM10, the RMSE ranged from 6.8 and $9.1 \mu \mathrm{g} / \mathrm{m}^{3}$ for $\mathrm{D}+0$ of the forecast, which is well below the key performance indicator defined at 18 $\mu \mathrm{g} / \mathrm{m}^{3}$. The corresponding bias is between -0.3 and $-0.1 \mu \mathrm{g} / \mathrm{m} 3$, and spatial correlation ranges from 0.71 to 0.75 . The precise definition of those statistical scores is available at https://regional.atmosphere.copernicus.eu/doc/USER_GUIDE_VERIFICATION_STATISTICS.pdf.

We will add a table with such statistics for all pollutants in the Supplement of the revised manuscript. However, we would like to underline that our study is based on the statistical analysis of the changes between 3 periods (before, during, and after the lockdown) in the differences "observed concentrations - expected concentrations" rather than on the comparison between observations and forecasts themselves.

3. There are not many published peer reviewed papers on the effect of lockdown measures on air pollution yet. We list changes observed in Brazil, China, and France in the introduction. Since the MS was submitted, a couple of studies were published for Spain (Madrid, Barcelona) and Europe as a whole (doi.org/10.1016/j.scitotenv.2020.140353, doi.org/10.5194/acp-2020-995, doi.org/10.5194/acp-2020-1171), the results of which will be mentioned in the revised version of our manuscript.

4. Line 19: we will specify in the abstract that we deal with ground-level concentrations.

5. Line 80 specifies that "total column-integrated NO2 tropospheric concentrations over northern Italy" were derived "using Sentinel-5p data".

6 . There was actually a desert dust outbreak reaching the measurement locations on March 28-29th (during the lockdown). This outbreak affected 2 days amongst 56 (8 weeks). We will compute statistics excluding these 2 days and state in the MS if this 
leads to any significant difference or not.

7. CAMS-Ensemble forecast made use of "anthropogenic emissions data based on 2011 emission inventories until June 2019, and on 2016 emission inventories afterwards" to stay up-to-date. This was not our own choice. 2019 forecast and observed data are used only to correct for seasonal changes in the difference between forecasts and observations. The way we calculate expected concentrations is independent from the change in emission inventories used by the CAMS-Ensemble model between 2019 and 2020.

8. The observation data from Ispra are 24-hr averages of measurements performed at a single location. Adding standard deviations would not be relevant. The observation data from Milan are averages from 3 urban background sites for all variables but PM10 (2), and standard deviations would not make sense. We would be glad to know if Referee \#2 retains useful to include error bars showing min and max values among the 3 (2) sites.

9. Unfortunately, there is no VOC data available for Ispra from March 8th to July 2nd, 2020 , because only fully automatic measurements could be carried out during the lockdown. Additional information based on data from previous years will be included in the manuscript to assess the $\mathrm{O} 3$ production chemical regime at this location.

10. Such an experiment of dramatically reducing NOx emissions in the whole of northern Italy never occurred before. Indeed, traffic limitation measures aiming at reducing wintertime particulate pollution peak at most affect half of the vehicles, and urban areas only. It is therefore difficult to compare what happened during the COVID lockdown with any previous situation.

Interactive comment on Atmos. Chem. Phys. Discuss., https://doi.org/10.5194/acp-2020-755, 2020. 
NOx emissions in Italy (2015)

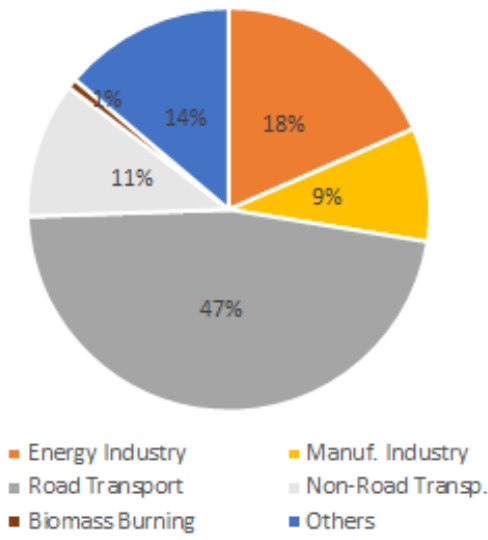

VOC emissions in Italy (2015)

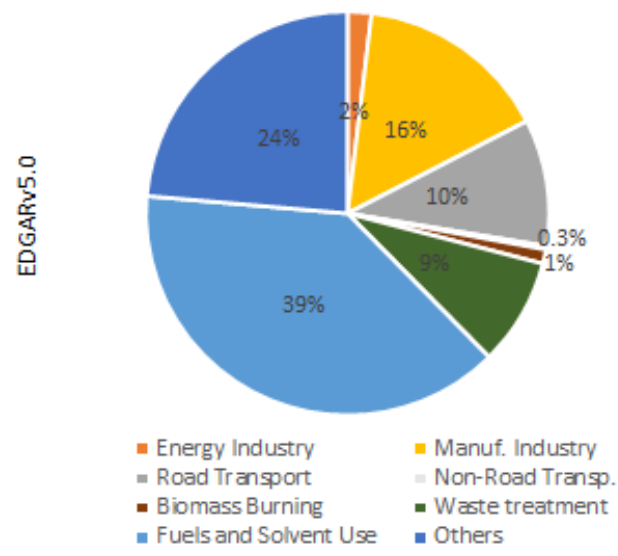

Fig. 1. 